\title{
Dexamethasone Inhibits Maturation and Alters Function of Monocyte-Derived Dendritic Cells from Cord Blood
}

\author{
ELSIE S. MAINALI, TAKESHI KIKUCHI, AND JOHN G. TEW \\ Department of Pediatrics [E.S.M.], VA Commonwealth University Health System, Richmond, Virginia \\ 23298; and Department of Microbiology and Immunology [T.K., J.G.T.], VA Commonwealth University, \\ Richmond, Virginia 23298
}

\begin{abstract}
ABS
Critically ill infants are treated with dexamethasone (Dx) and
other glucocorticoids to reduce inflammation and to promote
lung and cardiac function. The neonatal immune system is
immature, so neonatal dendritic cells (DCs) might be especially
sensitive to glucocorticoid-mediated immunosuppression. To test
this, we compared Dx treatment of monocyte-derived DCs from
cord (CB) and adult blood (AB). Dx decreased CD1a levels on
both AB and CB DCs. CB-treated cells also exhibited decreased
expression of CD83 and increased expression of CD14, alter-
ations not observed in AB DCs. Characteristic immature endo-
cytic activity was sustained and enhanced in Dx-treated CB DCs,
whereas AB DCs matured normally. Maintenance of endocytosis
corresponded with CD14 expression. Dx markedly increased CB
DC IL-10, a T cell helper 2 (Th2)-preferential cytokine, while
reducing IL-12, a counterbalancing Th1 cytokine. AB DCs were
also affected, but increases in IL-10 and decreases in IL-12 were
more modest. Dx treatment also inhibited DC-induced T cell
proliferation, but CB DCs were inhibited more. In short, neonatal
\end{abstract}
Cytokines that are produced by the placenta dampen allograft rejection and are thought to enhance the intrauterine survival of the fetus (1). This immune attenuation tends to direct an immune response toward $\mathrm{T}$ helper (Th) 2 and to weaken development of Th1-mediated immunity in newborns (2). The full range of adaptive immunity is gradually acquired through exposure to antigens that induce Th1 $\mathrm{T}$ cell responses and the development of a balanced immune system. When mothers go into premature labor, glucocorticoids, such as dexamethasone (Dx), are widely prescribed to enhance fetal lung maturation (3). In addition, glucocorticoids are used postnatally to reduce lung inflammation and to improve car-

Received May 26, 2004; accepted October 13, 2004

Correspondence: Elsie S. Mainali, M.D., Ph.D., Department of Pediatrics, VCUHS, 401 North 12th street, Room 6-500, Richmond, VA 23298; e-mail: emainali@hsc.vcu.edu.

This study was funded by the A.D. Williams Trust Foundation and Feamster Foundation Trust Fund, VA Commonwealth University. Flow cytometry work was supported in part by National Institutes of Health Grant P30 CA16059.

DOI: 10.1203/01.PDR.0000157677.72136.09
DCs seemed to be especially sensitive to the immunosuppressive effects of Dx as indicated by altered phenotype, endocytic function, ability to stimulate T cells, and cytokine shift favoring Th2. These alterations in DC function are consistent with an increased risk for certain infections and atopic diseases. (Pediatr Res 58: 125-131, 2005)

AB, adult blood
CB, cord blood
DC, dendritic cells
Dx, dexamethasone
GM-CSF, granulocyte-macrophage colony-stimulating factor
LPS, lipopolysaccharides
MFI, mean fluorescence intensity
MLR, mixed lymphocyte reaction
Th, T cell helper

diovascular status in critically ill infants (4). Dx-treated infants have alterations in immune responsiveness as indicated by significantly reduced antibody responses after immunization (5); increased infections (6); multiple short-term adverse complications such as intestinal perforation (7-9), hypertension, and hypertrophic cardiomyopathy $(10,11)$; and long-term effect such as increased risk for airway hyperresponsiveness and decreased pulmonary function $(12,13)$.

Several in vitro studies that were designed to identify the stage of maturation of each immune cell lineage in normal newborns have revealed that neonate naïve $\mathrm{T}(14-16)$ and $\mathrm{B}$ $(17,18)$ cells are as competent as their adult counterparts. However, neonatal dendritic cells (DCs) are functionally less competent (19) and less capable of secreting cytokines that are essential for development of Th1 responses and balanced immunity (20). DCs are the only professional antigenpresenting cells that are capable of priming neonatal naïve $\mathrm{T}$ cells for productive immune responses (21-23). This unique ability suggests that DCs play a vital role in the establishment 
of adaptive immunity in neonates. Little is known about the developmental biology of neonatal DCs. Moreover, studies on the effect of glucocorticoids such as Dx on neonatal DCs have not been done.

Studies in the murine system show that glucocorticoids inhibit DC maturation (24) and decrease DC function by reducing the expression of adhesion molecules CD11a, CD43, CD54, and CD90 and co-stimulatory molecules CD80 and CD86. DCs that express this immature phenotype have diminished ability to prime Th cells and to enhance the formation of T regulatory cells (25-27). Studies using human DCs derived from adult peripheral blood (AB) indicate that glucocorticoids 1) retard DC differentiation and maturation $(28,29)$ in a doseand length-of-exposure-dependent manner (30-32); 2) decrease expression of co-stimulatory molecules $(29,32) ; 3)$ alter DC functioning by increasing endocytosis, increasing secretion of IL-10 $(28,29,33,34)$ while decreasing IL-12 (29); and 4) weaken the immunostimulatory capacity of DCs in antigen presentation to autologous $\mathrm{T}$ cells in mixed lymphocyte reactions $(29,30,35,36)$.

The murine and adult blood studies described above and the immaturity of the neonatal immune system prompted us to reason that neonatal DCs might be particularly sensitive to the immunosuppressive effects of glucocorticoids. To begin testing this hypothesis, we compared the effects of Dx on monocytederived DCs from neonatal cord blood (CB) and AB. We found that Dx inhibited the expression of DC phenotypic markers and modulated differentiation of DCs from CB but did not affect DCs from $\mathrm{AB}$ to the same extent. Dx also altered cytokine production, causing a surge of IL-10 secretion and a simultaneous decline of IL-12 secretion, thus dramatically altering the ratio of IL-10 to IL-12. Remarkably, Dx seemed to induce CB monocytes to retain their lineage and differentiate toward macrophage-like cells with increased endocytic ability and reduced ability to stimulate $\mathrm{T}$ cells. In short, the data suggest that the treatment of neonates with Dx may alter DC function, leading to a reduction of $\mathrm{T}$ cell priming and a cytokine milieu biased toward Th2. This alteration in the neonatal immune system would be consistent with the increased risk for certain infections and the early manifestation of reactive airway diseases that may evolve into atopic diseases such as asthma $(12,13)$.

\section{METHODS}

Cell culture and Dx treatment. After informed consent in accordance with institutional review board standards, $\mathrm{CB}$ was collected into a sterile collection bag and processed within $24 \mathrm{~h}$. Mononuclear cells were separated from red cells by sedimentation with a solution of hydroxyethyl starch followed by density centrifugation over Histopaque-1077 (Sigma Chemical Co., St. Louis, $\mathrm{MO})$ to enrich the mononuclear cells. AB was collected by vena puncture, and buffy coat cells were separated. The cells that adhered after $1 \mathrm{~h}$ of incubation at $37^{\circ} \mathrm{C}$ (monocyte lineage cells) were cultured for $7 \mathrm{~d}$ in RPMI 1640 and $10 \%$ FCS enriched with $800 \mathrm{U} / \mathrm{mL}$ recombinant human granulocyte-macrophage colony-stimulating factor (GM-CSF) and $80 \mathrm{ng} / \mathrm{mL}$ recombinant human IL-4 (R \& D, Minneapolis, MN). For maturing DCs, $20 \mathrm{ng} / \mathrm{mL}$ lipopolysaccharide (LPS; Sigma Chemical Co.) was added to the culture at day 5. Dx (Sigma Chemical Co.) at $10^{-6} \mathrm{M}$ was added to half of the cells during the differentiation phase, during the first $5 \mathrm{~d}$ of culture $\left(\mathrm{Dx}^{0-5}\right)$, or during the last $2 \mathrm{~d}$ of culture $\left(\mathrm{Dx}^{5-7}\right)$. The concentration of $10^{-6} \mathrm{M}$ Dx was chosen on the basis of previous murine and adult peripheral blood in vitro studies $(30,37)$, and our preliminary studies, in which we found that $10^{-5} \mathrm{M}$ was lethal, whereas $10^{-8}$ and $10^{-9} \mathrm{M}$ had no effect on DC development. The culture media and reagents used were tested by the manufacturers, who indicate that their products are endotoxin-free.

Phenotypic marker analyses. Cells were incubated with the following mouse anti-human fluorescent MAb (Pharmingen, San Diego, CA) reactive with CD1a, CD83, CD86, CD14, or HLA-DP/Q/R (HLA-II) or with isotypematched antibodies as controls for $60 \mathrm{~min}$ at $4^{\circ} \mathrm{C}$. Flow cytometry data were acquired using an Epics-XL-MCL flow cytometer (Coulter, Fullerton, CA). A subpopulation was defined for its high concentration of cells that express DC markers and analyzed for each marker percentage expression and mean fluorescence intensity (MFI) using WinMDI software.

Test of endocytic function via uptake of soluble protein. The ability of cells to endocytose and catalyze the self-quenched green Bodipy dye conjugate of BSA (DQ-BSA; Molecular Probes, Eugene, OR), which involves clathrincoated pits, was used to measure endocytic function $(38,39)$. Cells were incubated with DQ-BSA $(10 \mu \mathrm{g} / \mathrm{mL}$ in RPMI 1640 that contained $10 \%$ fetal bovine serum) for $1 \mathrm{~h}$ at $37^{\circ} \mathrm{C}$, and controls were incubated on ice. The cells then were washed twice with PBS that contained $0.1 \% \mathrm{NaN}_{3}$ and $0.1 \%$ BSA (Sigma Chemical Co.) and incubated with phycoerythrin-labeled anti-CD14. Doubly labeled cells indicated endocytically active cells bearing the CD14 phenotype.

Cytokine determinations. Supernatant media from all cultures were collected after $5 \mathrm{~d}$. Cells were resuspended in media with LPS $(20 \mathrm{ng} / \mathrm{mL})$ to stimulate IL-10 or IL-12 and incubated for $2 \mathrm{~d}$ more according to the protocol described above. Supernatants from the cultures with or without Dx were collected at the end of total $7 \mathrm{~d}$ and frozen at $-80^{\circ} \mathrm{C}$ until assayed. Cytokine concentrations in the culture supernatants were assayed with commercial cytokine ELISA kits for human IL-10 and IL-12 according to the manufacturer's instructions (Amersham, Piscataway, NJ). The sensitivity of assays was $<3 \mathrm{pg} / \mathrm{mL}$ for IL-10 and IL-12. All cytokine assays were carried out in duplicate.

T cell proliferation assays. $\mathrm{T}$ cells from $\mathrm{CB}$ or $\mathrm{AB}$ were prepared at the time of blood processing by separating $\mathrm{CD}^{+}$cells from the nonadherent cells using CD3 cell enrichment magnetic beads (Miltenyi Biotec, Auburn, CA). Harvested $\mathrm{T}$ cells were cryopreserved according to an established protocol until used in the assay (40). Adherent CB or AB cells, cultured with recombinant human GM-CSF and recombinant human IL-4 for $5 \mathrm{~d}$ and LPS for an additional $2 \mathrm{~d}$, with or without Dx treatment, were transferred to a 96-well round-bottom tissue culture plate (Corning, NY) and irradiated with a 3000-rad ${ }^{137} \mathrm{Cs}$ source. The stimulator cells were used at 103,104 , or 105 cells per well, in triplicate wells, and incubated with the syngeneic [autologous mixed lymphocyte reaction (auto-MLR)] or allogeneic (allo-MLR) CB or AB T cells at 105 cells per well for $5 \mathrm{~d}$. The cultures were pulsed with $1 \mu \mathrm{Ci} /$ well [3H]TdR (NEN, Boston, MA) for the last $18 \mathrm{~h}$, then harvested and counted for radioactivity.

Statistical analysis. All experiments in this study were conducted three or more times. Experiments were typically set up with three replicates, and when variability was a problem, power calculations were done to determine the number of replicates needed. The patterns within experiments were reproducible, although the magnitude of responses between experiments could be larger, which prompted us to illustrate some results using individual experiments rather than pool data from multiple experiments. Comparison of the mean of two groups of treatment (nontreated versus treated with Dx) was examined by two-tailed $t$ test at a significance level of $p \leq 0.05$. All values were expressed as mean $\pm \mathrm{SD}$ unless otherwise stated.

\section{RESULTS}

Comparison of DC phenotypic marker expression by monocyte-derived $\mathrm{DCs}$ from $\mathrm{AB}$ and $\boldsymbol{C B}$. After $5 \mathrm{~d}$ of culture with GM-CSF and IL-4, a subpopulation of both CB- and AB-derived monocytes expressed the dendritic cell differentiation markers CD1a and CD83 in similar percentages and MFI. A representative example is shown in Fig. 1 (compare $A$, row 1 versus 2 and the means from three to nine individual experiments summarized in Table 1). Note that these markers were expressed even more robustly by both $\mathrm{CB}$ and $\mathrm{AB}$ cells after addition of LPS during the last $2 \mathrm{~d}$ of culture to stimulate maturation (Fig. 1B, Table 1). The percentage expression of the functional markers CD86 and HLA-II in CB- and AB-derived cells was very similar (Fig. 1, Table 1). The macrophage marker CD14 was higher at day 5 on CB than AB cells (12.6 


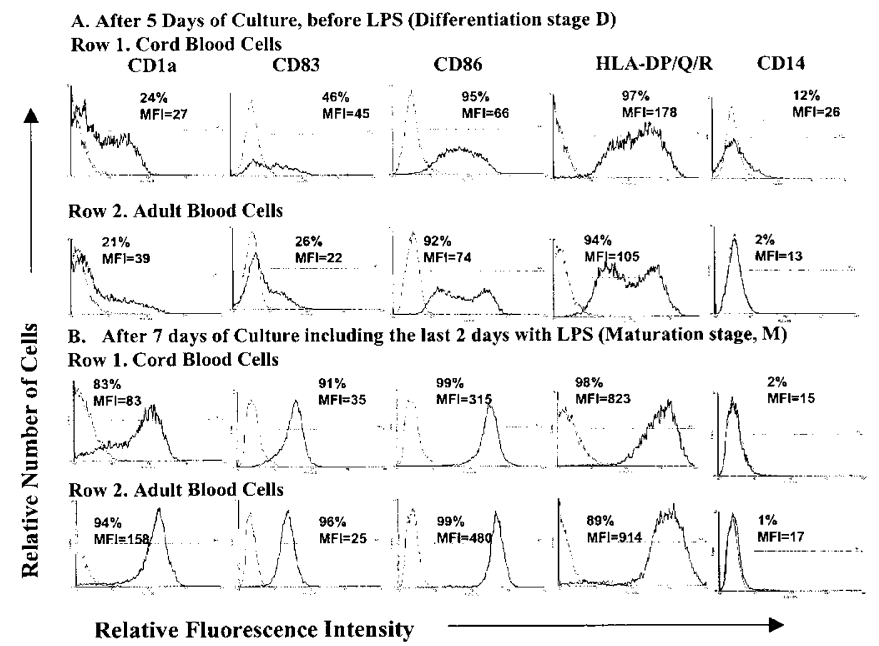

Figure 1. Expression of phenotypic markers on monocytes from $\mathrm{AB}$ and $\mathrm{CB}$ after differentiation with GM-CSF and IL-4 and maturation with LPS. The adherent cells from $\mathrm{CB}$ (row 1) or $\mathrm{AB}$ (row 2) were stimulated with GM-CSF and IL-4 for a $5 \mathrm{~d}$ differentiation period $\mathrm{D}(A)$ or continued in culture $2 \mathrm{~d}$ more with LPS as a maturation stimulus M $(B)$. Shown are overlay histograms of cell markers (thick lines) and isotype controls (thin lines), at the end of the differentiation stage, D, or at the end of the maturation stage, M. By the end of the differentiation stage $(A)$, the expression of phenotypic markers derived from $\mathrm{CB}$ cells was comparable to $\mathrm{AB}$ cells, with the exception of $\mathrm{CD} 14$, which was expressed significantly higher by the $\mathrm{CB}$ cells. The difference disappeared by the end of maturation stage $(B)$. Results are representative of three to nine different experiments.

versus $1.1 \% ; p<0.05$; Table 1 ), but this difference disappeared after $2 \mathrm{~d}$ of culture with LPS (2.1 versus $1.6 \%)$.

Dx treatment inhibited DC phenotypic marker expression and promoted CD14 expression. After CB cells were cultured with Dx for $5 \mathrm{~d}\left(\mathrm{Dx}^{0-5}\right)$, a significant decrease in the expression of CD1a was apparent (Fig. 2A, left). After $7 \mathrm{~d}$ of culture, expression of both DC differentiation markers CD1a and CD83 was significantly depressed by Dx treatment of $\mathrm{CB}$ cells whether they were treated during the differentiation stage $\left(\mathrm{Dx}^{0-5}\right)$ or only during the LPS maturation phase $\left(\mathrm{Dx}^{5-7}\right.$; Fig. $2 B$, left). Dx treatment of $A B$ cells during the differentiation phase, $\mathrm{Dx}^{0-5}$, tended to down-regulate CD1a and CD83 expression, but, after LPS stimulation, only CD1a was significantly reduced (Fig. 2, right). Expression of CD86 and HLA-II was not changed by Dx treatment of either $\mathrm{CB}$ or $\mathrm{AB}$ cells. The most dramatic Dx treatment effect was seen in the increased expression of the macrophage marker CD14 on CB-derived cells. After $5 \mathrm{~d}$ in Dx, the $\mathrm{CB}$ cells expressed 6-fold higher levels of CD14 than the untreated cells (Fig. 2A, left). The increased levels of CD14 persisted when the 5-d Dx treatment was followed by LPS stimulation and also were apparent when Dx was present only during LPS stimulation (Fig. 2B, left). When $\mathrm{Dx}$ was given to $\mathrm{AB}$ cells during the differentiation stage, a tendency toward increased $\mathrm{CD} 14^{+}$cells was apparent but did not reach statistical significance (Fig. 2A, right). When the $\mathrm{AB}$ cells were cultured for $2 \mathrm{~d}$ more with LPS and without Dx, CD14 expression decreased to control levels. There was no significant increase in AB-derived cell CD14 expression when Dx was added with LPS only during the maturation phase (Fig. $2 B$, right).
Dx enhanced endocytosis in CB-derived cells. The ability of DCs to take up and catalyze the self-quenched green Bodipy dye conjugates of BSA was assessed with flow cytometry and used as a measure of endocytic activity. Control CB DCs and AB DCs exhibited the expected high level of endocytic activity after $5 \mathrm{~d}$ of differentiation (Fig. 3A, D stage, white bar) and the expected decrease in endocytic activity after LPS induced maturation (Fig. 3A, M stage, white bar) (41). The addition of Dx to $\mathrm{CB}$ cells during the differentiation stage did not immediately affect the percentage of endocytosing cells (Fig. 3A, left, D stage, black bar), but, during the next $2 \mathrm{~d}$ of LPS stimulation without Dx, treated cells failed to exhibit the expected decrease in endocytic activity seen in the untreated cells (Fig. 3A, left, M stage, black bar). When Dx was added to $\mathrm{CB}$ cells only during the maturation stage, they also maintained significantly more endocytosing cells (Fig. $3 A$, left, M stage, hatched bar). In contrast, Dx-treated $\mathrm{AB}$ cells exhibited the decrease seen in untreated cells whether Dx treatment was during the differentiation stage or during the maturation phase (Fig. 3A, right, $\mathrm{M}$ stage, black and hatched bar).

The $\mathrm{Dx}^{0-5}$-treated $\mathrm{CB}$ and $\mathrm{AB}$ cells have the same percentage of cells taking up BSA as the untreated cells (Fig. 3A, D stage, white bar, $\mathrm{CB}$ and $\mathrm{AB}$ ), and each treated cell has approximately the same MFI as an untreated cell; that is, each treated cell takes up, on average, approximately as much BSA as an untreated cell (Fig. 3B, D stage, white bar, CB and AB). However, after LPS stimulation of the $\mathrm{Dx}^{0-5}$-treated CB cells, there was the unexpected increase in the percentage of endocytosing cells described above (Fig. $3 A$, CB, M stage, black bar), and there also was a remarkable increase in the amount of BSA that each cell was able to take up (Fig. 3B, CB, M stage). This phenomenon was not found in $\mathrm{AB}$ cells (Fig. 3, AB).

CD14 $^{+}$cells take up BSA. Because Dx-treated CB cells exhibited both increased CD14 expression and BSA uptake, we next determined whether the $\mathrm{CD} 14^{+}$cells were responsible for the increased BSA uptake by examining the expression of CD14 of cells that contained high levels of BSA. CB cells that were treated with $\mathrm{Dx}^{0-5}$ showed increases of CD14 ${ }^{+}$cells, and these same cells endocytosed BSA. This was apparent both at the end of the differentiation stage, D, and at the end of the maturation stage, M (Fig. 4, CB, black bar). This Dx effect on CB cells was not seen when Dx was added only during the maturation stage $\left(\mathrm{Dx}^{5-7}\right.$; Fig. 4, CB, hatched bar). Treated adult blood cells exhibited the same pattern of uptake by $\mathrm{CD} 4^{+}$cells as cord blood cells, but the results were less dramatic (Fig. 4, AB).

$D x$ treatment during differentiation promoted $I L-10$ and inhibited IL-12 secretion. During the last $2 \mathrm{~d}$ of culture, CB cells and $\mathrm{AB}$ cells secreted similar levels of IL-10 and IL-12, with IL-10 to IL-12 ratios of 6:1 and 5:1, respectively (Fig. 5, white bars for $\mathrm{CB}$ and $\mathrm{AB}$ ). However, $\mathrm{Dx}^{0-5}$-treated $\mathrm{CB}$ cells secreted significantly higher amounts of IL-10 and lower amounts of IL-12, so the ratio of IL-10 to IL-12 changed from 6:1 to 6219:1 (Fig. 5 left, black bars). In contrast, Dx treatment during the last $2 \mathrm{~d}$ (Fig. 5 left, hatched bars) of the 7-d culture period changed the ratio very little (from 6:1 to 9:1). Dx treatment of $\mathrm{AB}$ cell for $5 \mathrm{~d}$ also altered the IL-10 to IL-12 ratio but to a lesser extent than for CB cells: from 5:1 to 483:1 (Fig. 
Table 1. Comparison of phenotypic markers on monocyte-derived DCs from $C B$ and $A B$

\begin{tabular}{|c|c|c|c|c|c|}
\hline \multirow{2}{*}{$\begin{array}{l}\text { Days of culture } \\
\text { (d) }\end{array}$} & \multicolumn{5}{|c|}{$\%$ Cells exhibiting the marker $($ mean $\pm \mathrm{SD})$} \\
\hline & CD1a & CD83 & CD86 & HLA-II & CD14 \\
\hline $5 \mathrm{~d} \mathrm{CB}(n=5)$ & $36.2 \pm 15.8$ & $32.4 \pm 12.5$ & $84.1 \pm 10.5$ & $98.1 \pm 1.2$ & $11.8 \pm 1.7$ \\
\hline $7 \mathrm{~d} \mathrm{CB}(n=10)$ & $52.5 \pm 26.1$ & $77.2 \pm 14.9$ & $87.9 \pm 10.3$ & $97.5 \pm 2.4$ & $2.1 \pm 1.8$ \\
\hline $7 \mathrm{~d} \mathrm{AB}(n=12)$ & $64.9 \pm 27.7$ & $75.5 \pm 22.6$ & $95.1 \pm 5.4$ & $96.4 \pm 3.4$ & $1.6 \pm 1.4$ \\
\hline
\end{tabular}

$* p<0.05(5-\mathrm{d} \mathrm{CB}$ vs 5-d AB).

CORD BLOOD (CB)

A. Before LPS ( $\mathrm{No} \mathrm{Dx}^{0-5} ; \mathrm{aDx}^{0-5}$ ): Differentiation stage

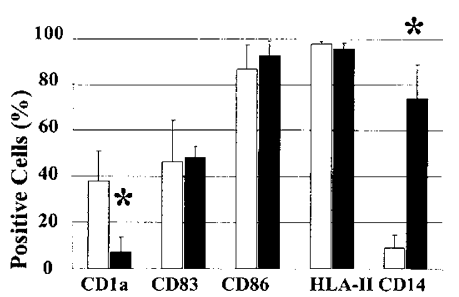

B. After LPS ( $\square \mathrm{No} \mathrm{Dx}^{0-7}, \square \mathrm{Dx}^{0-5}$, and
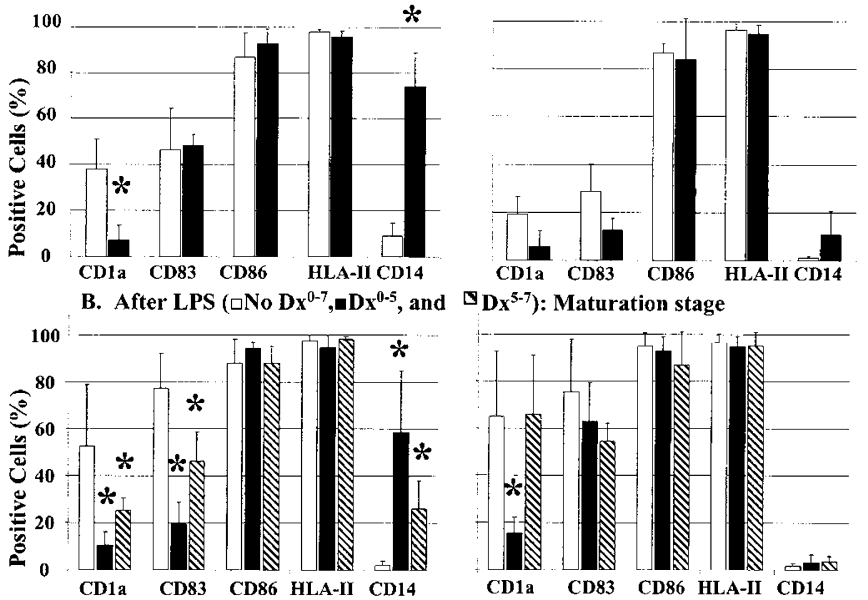

$\left.\nabla_{D x^{5-7}}\right)$ : Maturation stage

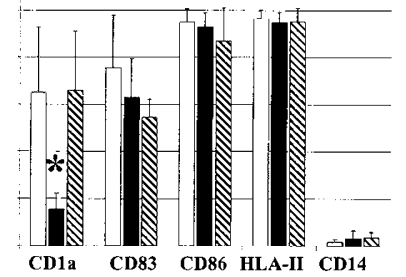

Figure 2. Effect of Dx on the expression of phenotypic markers characteristic of DCs. (A) Influence of Dx on the expression of DC markers and CD14 on CB- and AB-derived DCs $(\square)$ compared with no Dx treatment $(\square)$. Note the alterations of CD1a and CD14 expression in Dx-treated cells derived from CB and $\mathrm{AB}$ at the end of the 5-d differentiation period $\mathrm{D}$. $(B)$ The percentage of CB- and AB-derived cells expressing DC markers and CD14 at the end of the maturation stage $\mathrm{M}$. The alteration of CD1a, CD83, and CD14 expression in Dx-treated cells derived from CB was statistically significant, whereas only CD1a was significantly suppressed in AB-derived cells. Values are expressed as mean percentages $\pm \mathrm{SD}$ of six to nine different experiments. ${ }^{*} p<0.05$ (Dx treatment vs no Dx treatment control).

5, right, black bars). The ratio for Dx treatment of $\mathrm{AB}$ cells during maturation $\left(\mathrm{Dx}^{5-7}\right.$; Fig. 5 right, hatched bars) also increased slightly (from 5:1 to 18:1). Thus, although the cytokine secretions for both $\mathrm{CB}$ and $\mathrm{AB}$ cells changed as a result of Dx treatment, the ratio of changes during differentiation were much greater for $\mathrm{CB}$ cells than for $\mathrm{AB}$ cells. In the absence of LPS during maturation, neither IL-10 nor IL-12 was detected at the end of the 7-d incubation period, indicating that cytokine secretion was LPS dependent (data not shown).

Comparison of the inhibitory effect of Dx on the ability of $A B$ and $C B$ DCs to induce auto- and allo-T cell proliferations. DCs have a remarkable ability to stimulate auto- and allo-MLRs. On average, CB DCs had $\sim 30-50 \%$ of the ability of adult-derived DCs to stimulate auto- and allo-MLRs. Dx treatment further diminished their ability to stimulate autologous MLR (50\%) and allo-MLR ( 30\%; Fig. 6A). Similarly, Dx inhibited auto- and allo-MLRs of AB DCs, but the inhibition was less consistent and not as dramatic (Fig. 6B).

\section{DISCUSSION}

Despite their known immunosuppressive activities, glucocorticoids, particularly Dx, are widely prescribed in the

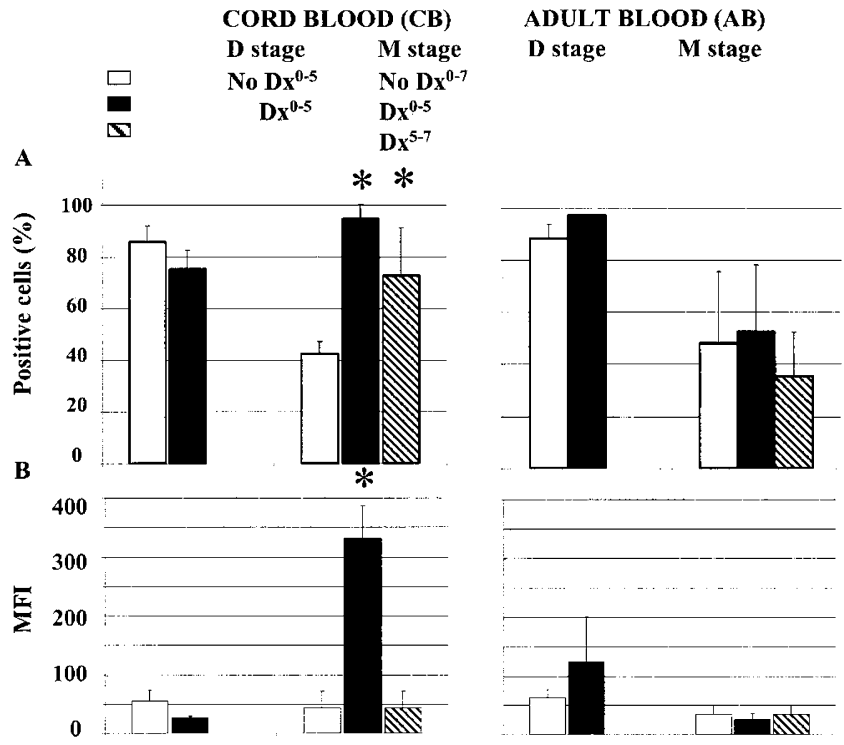

Figure 3. Effect of Dx on DC endocytic activity at the end of the 5-d differentiation period and after the 2-d maturation period. (A) The percentage of DCs that were treated with Dx from days $0-5\left(\mathrm{Dx}^{0-5} ; \mathbf{\square}\right)$ and untreated $(\square)$ taking up green Bodipy dye labeled BSA or with Dx from days $5-7\left(\mathrm{Dx}^{5-7} ;\right)$. Dx-treated cells from CB showed significantly higher ability to take up BSA, suggesting the persistence of immature function. $(B)$ The MFI of cells taking up labeled BSA as explained above. At the end of the 7-d culture, CB cells that were treated with Dx on days 0 to 5 ( $\square$ ) showed significantly high fluorescent intensity, suggesting that Dx treatment during the differentiation stage caused not only maintenance of BSA uptake but also ability to endocytose a higher amount of BSA. Values are expressed as means \pm SD of three to six individual experiments. ${ }^{*} p<0.05$ (Dx treatment $v s$ no Dx treatment control).

perinatal period to enhance fetal lung maturation (42), reduce lung inflammation (3), and improve cardiovascular function (4). Glucocorticoids have been shown to inhibit maturation of murine DCs (24) by reducing the expression of adhesion molecules and co-stimulatory molecules, resulting in diminished priming and activation of specific $\mathrm{T}$ cells $(25,26)$ or up-regulation of IL-10 secreting T regulatory cells (27). Cultured adult human cells that are treated with glucocorticoids decrease expression of DC co-stimulatory molecules $(29,32)$, retard DC differentiation and maturation (29-32), alter DC endocytic function, and weaken DC immunostimulatory capacity in antigen presentation to autologous $\mathrm{T}$ cells $(29,30,33,35,36)$. Our study confirmed most of these effects on AB DCs but, more important, showed a greater impact of Dx on monocyte-derived DCs from neonatal than adult blood cells. Nevertheless, it should be appreciated that cell-to-cell contact and mediators in the tissue milieu might influence the effect of Dx on DCs in vivo. 


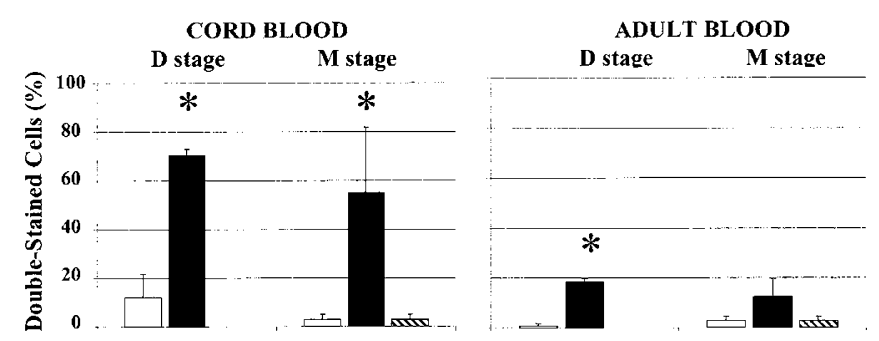

Figure 4. Correlation of increased endocytosis with expression of CD14. Cells were treated as explained in Fig. 3 but were double-labeled with green Bodipy dye-conjugated BSA and anti-CD14-phycoerythrin. Bars indicate percentage of CD14 ${ }^{+}$cells taking up BSA: untreated $(\square)$ or Dx treated on days $0-5\left(\mathrm{Dx}^{0-5 \mathrm{D}} ; \mathbf{\square}\right)$ and $\mathrm{Dx}$ treated on days $5-7\left(\mathrm{Dx}^{5-7} ;\right)$. Dx significantly increased the percentage of CB-derived cells that expressed CD14 and were able to endocytose BSA at the end of either the differentiation stage or the maturation stage. The same trend was observed in AB-derived cells, but differences reached statistical significance only at the end of differentiation stage. Values are expressed as means $\pm \mathrm{SD}$ of three to six individual experiments. ${ }^{*} p<0.05$ (Dx treatment $v s$ no Dx treatment control).

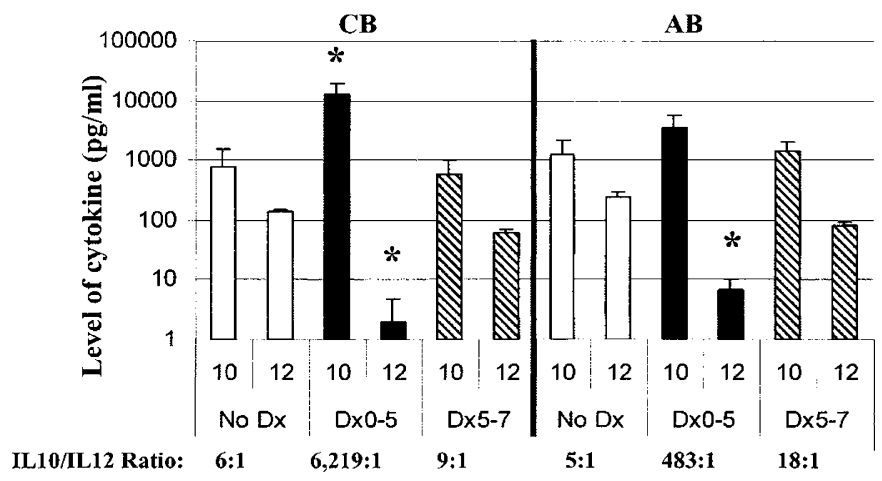

Figure 5. Dx enhanced IL-10 and inhibited IL-12 secretion by DCs. DCs that were generated from monocytes of either $\mathrm{CB}$ (left) or $\mathrm{AB}$ (right) were incubated for $7 \mathrm{~d}$ with Dx for $0 \mathrm{~d}$ (no Dx; $\square$ ), 0-5 d ( $\left.\mathrm{Dx}^{0-5} ; \square\right)$, or $5-7 \mathrm{~d}$ $\left(\mathrm{Dx}^{5-7}\right.$; ) with the addition of $20 \mathrm{ng} / \mathrm{mL}$ LPS to induce maturation on days 5-7. At the end of the 7-d culture, the accumulated IL-10 (left bar of each treatment pair) and IL-12p70 levels (right bar of each treatment pair) were determined in culture supernatant fluids by ELISA. Dx exposure during the differentiation stage $\left(\mathrm{Dx}^{0-5}\right)$ increased the ratio of IL-10 to IL-12p70 level secreted by CB-derived cells $\sim 13$ times more than AB. Data are shown as means \pm SD of three independent experiments from three different individuals. ${ }^{*} p<0.05$ (Dx treatment $v s$ no Dx treatment control).

The phenotype of CB-DCs was very similar to that of $\mathrm{AB}$ DCs (Fig. 1, Table 1). One noticeable difference was that CB-derived cells expressed more CD14 than AB-derived cells after $5 \mathrm{~d}$ of culture, indicating the presence of more macrophage-like cells. However, both CB- and AB-derived cells expressed negligible CD14 after LPS stimulation for $2 \mathrm{~d}$. Clearly, the adherent monocytes of both $\mathrm{CB}$ and $\mathrm{AB}$ have similar differentiation and maturation potentials.

Dx treatment decreased expression of the DC differentiation markers CD1a and CD83 of CB-derived cells even when treated just during the maturation stage. This result suggests that Dx may be able to alter DC maturation in a neonate even after normal differentiation. In contrast, AB DCs were resistant to Dx effects during the maturation stage. The expression of CD86 and HLA on DCs was not changed by Dx treatment of $\mathrm{CB}$ or $\mathrm{AB}$ cells, suggesting that these markers that are crucial for antigen presentation are more resistant to Dx-mediated suppression.

In contrast to the Dx-mediated depression of DC CD1a and CD83, the expression of the macrophage marker CD14 in cells derived from $\mathrm{CB}$ was increased relative to the untreated control both when Dx was added during the differentiation stage and when it was added only during the maturation stage. The results suggest that Dx is instrumental in sustaining macrophage-like cells in CB cultures, and these data are consistent with the hypothesis that Dx is a genomic-affecting steroid (43). Canning et al. (44) reported a similar increase of CD14 expression when Dx was added during the differentiation stage in a human adult monocyte/DC culture, although Dx effect during the maturation stage was not studied.

Mellman et al. (45) found that most newly differentiated DCs from $\mathrm{AB}$ take up large quantities of $\mathrm{Ag}$, but this high level of endocytic ability decreases as LPS stimulates DCs to switch from $\mathrm{Ag}$ uptake to $\mathrm{Ag}$ processing during maturation. We also found this decreased DC endocytosis after maturation in both untreated AB- and CB-derived cells (Fig. 3A). In contrast, Dx treatment maintained the high percentage of $\mathrm{CB}$ cells that were capable of taking up Ag during maturation, whether Dx was present during the differentiation or maturation stages. When given during the differentiation stage, Dx not only increased the percentage of $\mathrm{CB}$ cells that can take up $\mathrm{Ag}$ but also dramatically increased the amount of Ag that each cell takes up during stimulation with LPS (Fig. 3B, left). Dx treatment during differentiation seems to be causing LPS-stimulated cells to maintain endocytic activity, rather than allowing normal DC maturation development from $\mathrm{Ag}$ uptake to $\mathrm{Ag}$ processing and presentation. This increase of cells that are capable of taking up $\mathrm{Ag}$ as a result of Dx treatment that was seen with CB cells was not seen with $\mathrm{AB}$ cells (Fig. 3), suggesting, again, that $\mathrm{CB}$ cells are more susceptible to Dx treatment, especially during the differentiation stage of DC development.

The persisting $\mathrm{CD} 14^{+}$cells after Dx treatment were responsible for two thirds of the increase observed in $\mathrm{Ag}$ uptake at the end of the differentiation stage, D, and almost all of the increase resulting by the end of the maturation stage, M (Fig. 4, CB). AB cells also responded to Dx treatment during differentiation with modestly increased endocytic CD14 ${ }^{+}$cells, concurring with the reported finding by Canning et al. (44); however, this increase was not retained during the maturation stage, $\mathrm{M}$ (Fig. 4, AB). In addition, the percentage of endocytic $\mathrm{CD} 14^{+} \mathrm{CB}$ cells was three times that of $\mathrm{AB}$ cells, reinforcing the notion that $\mathrm{CB}$ cells are more susceptible to Dx than $\mathrm{AB}$ cells.

After $7 \mathrm{~d}$ of culture in the absence of Dx, the levels of IL-10 and IL-12 produced by $\mathrm{CB}$ cells were comparable to those produced by $\mathrm{AB}$ cells (Fig. 5). In contrast, others have reported weak IL-12 secretion by $\mathrm{CB}$ compared with $\mathrm{AB}$ mononuclear cells (46-49), suggesting that the ability of CB DCs to secrete IL-12 may not be as good as AB DCs. The reasons for these differences are not understood but may relate to differences in the state of the DCs in the different cultures.

The observation that Dx-treated adult human monocytederived DCs showed up-regulation of IL-10, a Th2-preferential cytokine with comparable down-regulation of IL-12 secretion, 
a counterbalancing Th1 cytokine, confirms the work of others $(29,33,34,44)$. The observation that Dx added to CB cells during the differentiation stage dramatically amplified the modulation compared with $\mathrm{AB}$ cells extends previous observations (Fig. 5). This large-scale alteration in the IL-10 to IL-12 ratio would greatly favor induction of Th2 T cells. It has been reported that a reduced amount of IL-12 production during the perinatal period can be linked to a predominantly Th2 atopic manifestation in later life (49). Children who are exposed to glucocorticoids seem to be at increased risk for airway hyperresponsiveness and decreased pulmonary function $(12,13)$ with a tendency to develop asthma. Our findings that Dx treatment skews toward a Th2 cell milieu would be consistent with this observation and contribute to the concern that treatment of children who have asthma with steroids may further bias them toward Th2 immunity and ultimately predispose these children toward more atopic disease.

Both AB and CB DCs could stimulate auto- and allo-MLRs (Fig. 6), although $\mathrm{CB}$ DCs were not as potent as $\mathrm{AB}$ DCs. Moreover, both $\mathrm{AB}$ and $\mathrm{CB}$ DCs were inhibited by Dx treatment, but the CB DCs were most affected. CB DCs were inhibited over all stimulator to $\mathrm{T}$ cell ratios studied, and inhibition was apparent during DC differentiation and maturation. The reduced ability of CB DCs to stimulate T cells when treated with Dx probably relates to a failure to differentiate as evidenced by the observed decrease in DC differentiation markers together with persistence of the monocyte marker CD14. Our data also showed an impressive surge of IL-10 secretion upon Dx treatment during the differentiation stage $\left(\mathrm{Dx}^{0-5}\right.$; Fig. 5). Because IL-10 is a known inhibitor of DC maturation (50), Dx may be inhibiting the DC maturation
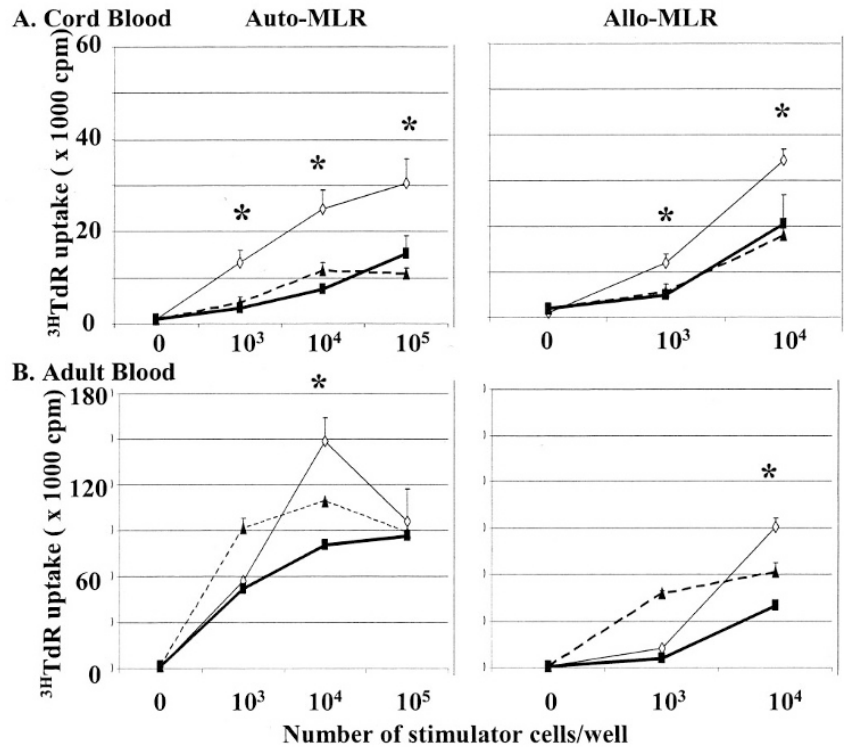

Figure 6. Effect of Dx on the ability of $\mathrm{CB}$ and $\mathrm{AB}$ monocyte-derived DCs to stimulate autologous and allogeneic T cell proliferation. Untreated DCs $(\diamond)$ are compared with those treated with Dx on days $0-5\left(\mathrm{Dx}^{0-5} ; \boldsymbol{\square}\right)$ and those treated with Dx on days $5-7\left(\mathrm{Dx}^{5-7} ; \mathbf{4}\right)$. Proliferation was determined by ${ }^{3 \mathrm{H}} \mathrm{TdR}$ uptake and was expressed as mean $\pm \mathrm{SD}$ of triplicate cultures. Dx suppressed the ability of CB-derived cells to stimulate autologous and allogeneic lymphocytes. Data are representative of three experiments of this type. ${ }^{*} p$ $<0.05$ (Dx treatment $v s$ no Dx treatment control). process through this pathway, thus contributing to the reduced induction of $\mathrm{T}$ cell proliferation by Dx-treated DCs.

\section{CONCLUSION}

In conclusion, this study documents the suppressive effects of Dx on the development and function of DCs of neonatal origin. Dx retards the expression of essential DC phenotypic markers, increases the number of $\mathrm{CD} 14^{+}$cells that are more efficient in endocytosis but less effective as antigen presenters, amplifies the secretion of Th2-biased IL-10, and decreases the induction of $\mathrm{T}$ cell proliferation. These findings suggest that treatment of neonates with Dx may adversely alter DC cell function, reduce naïve $T$ cell priming, deter the development of active immunity, and increase the potential for opportunistic infections such as candidiasis (51-53). Alteration of the IL-10 to IL-12 ratio that leads to a Th2 bias may hasten and perpetuate the manifestation of atopic diseases such as asthma. These clinical implications support a need for comparative studies with other glucocorticoids to determine whether glucocorticoids such as betamethasone, methylprednisolone, or prednisolone, which do not have the same gene-modifying effects as Dx (43), might provide the needed benefits to neonates without altering DC function.

Acknowledgments. We thank the house staff and nurses in the labor and delivery sections of the Department of Obstetrics and Gynecology, VCUMC, for CB collection, and Frances White and Julie Farnsworth for invaluable technical assistance in flow cytometry. We also thank Dr. Shirley K. DeSimone for editing the manuscript.

\section{REFERENCES}

1. Wegmann TG, Lin H, Guilbert L, Mosmann TR 1993 Bidirectional cytokine interactions in the maternal-fetal relationship: is successful pregnancy a TH2 phenomenon? Immunol Today 14:353-356

2. Guller S, LaChapelle L 1999 The role of placental Fas ligand in maintaining immune privilege at maternal-fetal interfaces. Semin Reprod Endocrinol 17:39-44

3. Banks BA, Macones G, Cnaan A, Merrill JD, Ballard PL, Ballard RA; North American TRH Study Group 2002 Multiple courses of antenatal corticosteroids are associated with early severe lung disease in preterm neonates. J Perinatol 22:101-107

4. Seri I, Tan R, Evans J 2001 Cardiovascular effects of hydrocortisone in preterm infants with pressor-resistant hypotension. Pediatrics 107:1070-1074

5. Robinson MJ, Campbell F, Powell P, Sims D, Thornton C 1999 Antibody response to accelerated Hib immunisation in preterm infants receiving dexamethasone for chronic lung disease. Arch Dis Child Fetal Neonatal Ed 80:F69-F71

6. Yeh TF, Lin YJ, Hsieh WS, Lin HC, Lin CH, Chen JY, Kao HA, Chien CH 1997 Early postnatal dexamethasone therapy for the prevention of chronic lung disease in preterm infants with respiratory distress syndrome: a multicenter clinical trial. Pediatrics 100:E3

7. Gordon PV, Young ML, Marshall DD 2001 Focal small bowel perforation: an adverse effect of early postnatal dexamethasone therapy in extremely low birth weight infants. J Perinatol 21:156-160

8. Stark AR, Carlo WA, Tyson JE, Papile LA, Wright LL, Shankaran S, Donovan EF, Oh W, Bauer CR, Saha S, Poole WK, Stoll BJ 2001 Adverse effects of early dexamethasone in extremely-low-birth-weight infants. National Institute of Child Health and Human Development Neonatal Research Network. N Engl J Med 344:95101

9. De Laet MH, Dassonville M, Johansson A, Lerminiaux C, Seghers V, Van den Eijnden S, Blum D, Vanderwinden JM 2000 Small-bowel perforation in very low birth weight neonates treated with high-dose dexamethasone. Eur J Pediatr Surg 10:323-327

10. Halliday HL, Ehrenkranz RA, Doyle LW 2003 Moderately early (7-14 days) postnatal corticosteroids for preventing chronic lung disease in preterm infants. Cochrane Database Syst Rev CD001144

11. Halliday HL, Ehrenkranz RA, Doyle LW 2003 Early postnatal ( $<96$ hours) corticosteroids for preventing chronic lung disease in preterm infants. Cochrane Database Syst Rev CD001146

12. Kennedy JD, Edward LJ, Bates DJ, Martin AJ, Dip SN, Haslam RR, McPhee AJ, Staugas RE, Baghurst P 2000 Effects of birthweight and oxygen supplementation on 
lung function in late childhood in children of very low birth weight. Pediatr Pulmonol 30:32-40

13. Pelkonen AS, Hakulinen AL, Turpeinen M 1997 Bronchial lability and responsive ness in school children born very preterm. Am J Respir Crit Care Med 156:11781184

14. Roncarolo MG, Bigler M, Ciuti E, Martino S, Tovo PA 1994 Immune responses by cord blood cells. Blood Cells 20:573-585

15. Risdon G, Gaddy J, Broxmeyer HE 1994 Allogeneic responses of human umbilical cord blood. Blood Cells 20:566-570

16. Harris DT 1994 Cord blood transplantation: implications for graft vs. host disease and graft vs. leukemia. Blood Cells 20:560-564

17. Splawski JB, Jelinek DF, Lipsky PE 1991 Delineation of the functional capacity of human neonatal lymphocytes. J Clin Invest 87:545-553

18. Ueno Y, Ichihara T, Hasui M, Maruyama H, Miyawaki T, Taniguchi N, Komiyama A 1992 T-cell-dependent production of IgG by human cord blood B cells in reconstituted SCID mice. Scand J Immunol 35:415-419

19. Petty RE, Hunt DW 1998 Neonatal dendritic cells. Vaccine 16:1378-1382

20. Trivedi HN, HayGlass KT, Gangur V, Allardice JG, Embree JE, Plummer FA 1997 Analysis of neonatal $\mathrm{T}$ cell and antigen presenting cell functions. Hum Immunol 57:69-79

21. Liu LM, MacPherson GG 1993 Antigen acquisition by dendritic cells: intestinal dendritic cells acquire antigen administered orally and can prime naive T cells in vivo. J Exp Med 177:1299-1307

22. Inaba K, Metlay JP, Crowley MT, Steinman RM 1990 Dendritic cells pulsed with protein antigens in vitro can prime antigen-specific, MHC-restricted T cells in situ. J Exp Med 172:631-640

23. Sornasse T, Flamand V, De Becker G, Bazin H, Tielemans F, Thielemans K, Urbain J, Leo O, Moser M 1992 Antigen-pulsed dendritic cells can efficiently induce an antibody response in vivo. J Exp Med 175:15-21

24. Matyszak MK, Citterio S, Rescigno M, Ricciardi-Castagnoli P 2000 Differentia effects of corticosteroids during different stages of dendritic cell maturation. Eur J Immunol 30:1233-1242

25. Sacedon R, Vicente A, Varas A, Jimenez E, Munoz JJ, Zapata AG 1999 Glucocorticoid-mediated regulation of thymic dendritic cell function. Int Immunol 11:12171224

26. Moser M, De Smedt T, Sornasse T, Tielemans F, Chentoufi AA, Muraille E, Van Mechelen M, Urbain J, Leo O 1995 Glucocorticoids down-regulate dendritic cell function in vitro and in vivo. Eur J Immunol 25:2818-2824

27. Dong X, Bachman LA, Kumar R, Griffin MD 2003 Generation of antigen-specific interleukin-10-producing T-cells using dendritic cell stimulation and steroid hormone conditioning. Transpl Immunol 11:323-333

28. Vieira PL, Kalinski P, Wierenga EA, Kapsenberg ML, de Jong EC 1998 Glucocorticoids inhibit bioactive IL-12p70 production by in vitro-generated human dendritic cells without affecting their T cell stimulatory potential. J Immunol 161:5245-5251

29. de Jong EC, Vieira PL, Kalinski P, Kapsenberg ML 1999 Corticosteroids inhibit the production of inflammatory mediators in immature monocyte-derived DC and induce the development of tolerogenic DC3. J Leukoc Biol 66:201-204

30. Piemonti L, Monti P, Allavena P, Sironi M, Soldini L, Leone BE, Socci C, Di Carlo V 1999 Glucocorticoids affect human dendritic cell differentiation and maturation. J Immunol 162:6473-6481

31. Demeure CE, Tanaka H, Mateo V, Rubio M, Delespesse G, Sarfati M 2000 CD47 engagement inhibits cytokine production and maturation of human dendritic cells. J Immunol 164:2193-2199

32. Verhoeven GT, Van Haarst JM, De Wit HJ, Simons PJ, Hoogsteden HC, Drexhage HA 2000 Glucocorticoids hamper the ex vivo maturation of lung dendritic cells from their low autofluorescent precursors in the human bronchoalveolar lavage: decreases in allostimulatory capacity and expression of CD80 and CD86. Clin Exp Immuno 122:232-240

33. Pedersen AE, Gad M, Walter MR, Claesson MH 2004 Induction of regulatory dendritic cells by dexamethasone and $1 \alpha, 25$-dihydroxyvitamin $\mathrm{D}(3)$. Immunol Lett 91:63-69
34. Mozo L, Suarez A, Gutierrez C 2004 Glucocorticoids up-regulate constitutive interleukin-10 production by human monocytes. Clin Exp Allergy 34:406-412

35. Rea D, van Kooten C, van Meijgaarden KE, Ottenhoff TH, Melief CJ, Offringa R 2000 Glucocorticoids transform CD40-triggering of dendritic cells into an alternative activation pathway resulting in antigen-presenting cells that secrete IL-10. Blood 95:3162-3167

36. Matasic R, Dietz AB, Vuk-Pavlovic S 1999 Dexamethasone inhibits dendritic cell maturation by redirecting differentiation of a subset of cells. J Leukoc Biol 66:909914

37. Pan J, Ju D, Wang Q, Zhang M, Xia D, Zhang L, Yu H, Cao X 2001 Dexamethasone inhibits the antigen presentation of dendritic cells in MHC class II pathway. Immunol Lett 76:153-161

38. Cherukuri A, Durack G, Voss EW Jr 1997 Evidence for hapten recognition in receptor-mediated intracellular uptake of a hapten-protein conjugate by murine macrophage. Mol Immunol 34:21-32

39. Weaver DJ Jr, Voss EW Jr 1998 Analysis of rates of receptor-mediated endocytosis and exocytosis of a fluorescent hapten-protein conjugate in murine macrophage: implications for antigen processing. Biol Cell 90:169-181

40. Donaldson SL, Miller GA, Rice PL, Ranney RR, Tew JG 1981 The maintenance of B-cell and T-cell function in frozen and stored human lymphocytes. J Clin Immunol $1: 106-112$

41. Mahnke K, Schmitt E, Bonifaz L, Enk AH, Jonuleit H 2002 Immature, but not inactive: the tolerogenic function of immature dendritic cells. Immunol Cell Biol 80:477-483

42. Mayani H, Lansdorp PM 1998 Biology of human umbilical cord blood-derived hematopoietic stem/progenitor cells. Stem Cells 16:153-165

43. Buttgereit F, Brand MD, Burmester GR 1999 Equivalent doses and relative drug potencies for non-genomic glucocorticoid effects: a novel glucocorticoid hierarchy. Biochem Pharmacol 58:363-368

44. Canning MO, Grotenhuis K, de Wit HJ, Drexhage HA 2000 Opposing effects of dehydroepiandrosterone and dexamethasone on the generation of monocyte-derived dendritic cells. Eur J Endocrinol 143:687-695

45. Mellman I, Turley SJ, Steinman RM 1998 Antigen processing for amateurs and professionals. Trends Cell Biol 8:231-237

46. Lee SM, Suen Y, Chang L, Bruner V, Qian J, Indes J, Knoppel E, van de Ven C, Cairo MS 1996 Decreased interleukin-12 (IL-12) from activated cord versus adult peripheral blood mononuclear cells and upregulation of interferon-gamma, natural killer, and lymphokine-activated killer activity by IL-12 in cord blood mononuclear cells. Blood 88:945-954

47. Joyner JL, Augustine NH, Taylor KA, La Pine TR, Hill HR 2000 Effects of group B streptococci on cord and adult mononuclear cell interleukin-12 and interferon-gamma mRNA accumulation and protein secretion. J Infect Dis 182:974-977

48. La Pine TR, Joyner JL, Augustine NH, Kwak SD, Hill HR 2003 Defective production of IL-18 and IL-12 by cord blood mononuclear cells influences the T helper-1 interferon gamma response to group B Streptococci. Pediatr Res 54:276-281

49. Prescott SL, Taylor A, King B, Dunstan J, Upham JW, Thornton CA, Holt PG 2003 Neonatal interleukin-12 capacity is associated with variations in allergen-specific immune responses in the neonatal and postnatal periods. Clin Exp Allergy 33:566572

50. Sallusto F, Lanzavecchia A 2002 The instructive role of dendritic cells on T-cell responses. Arthritis Res 4(suppl 3):S127-S132

51. Rowen JL, Atkins JT, Levy ML, Baer SC, Baker CJ 1995 Invasive fungal dermatiti in the $<$ or $=1000$-gram neonate. Pediatrics 95:682-687

52. Botas CM, Kurlat I, Young SM, Sola A 1995 Disseminated candidal infections and intravenous hydrocortisone in preterm infants. Pediatrics 95:883-887

53. Pera A, Byun A, Gribar S, Schwartz R, Kumar D, Parimi P 2002 Dexamethasone therapy and Candida sepsis in neonates less than 1250 grams. J Perinatol 22:204-208 